\title{
Research of Dynamic Response of Orthotropic Elastic Media under Harmonic Loads
}

\author{
Chunli Zhang ${ }^{1, a}$, Bo Wang ${ }^{2, b}$, Yanzhi Zhu ${ }^{3, c}$ and Shunli Xie ${ }^{3, d}$ \\ ${ }^{1}$ School of Water Conservancy \& Environment, Zhengzhou University, and School of Civil \\ Engineering and Architecture, Zhongyuan University of Technology, China \\ ${ }^{2}$ School of Water Conservancy \& Environment, Zhengzhou University, China \\ ${ }^{3}$ School of Civil Engineering and Architecture, Zhongyuan University of Technology, China

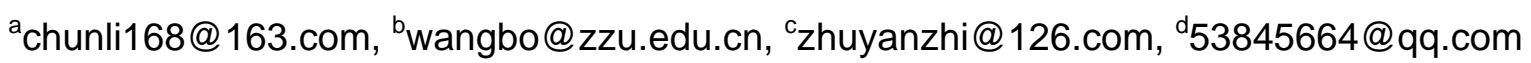

Keywords: Orthotropic, plane strain, dynamic response, Fourier transforms.

Abstract. Two-dimensional dynamic response of orthotropic media under rectangular coordinate system was studied. With displacement component of the basic unknown quantity, the dynamic equations were drawn for orthotropic media, and the dynamic responses in time domain for orthotropic elastic media under harmonic loads was derived by means of Fourier transform. Vertical displacement curves of the calculated point due to different depth, different soil parameters and load frequencies are researched.

\section{Introduction}

Generally, the soil is in the plane strain state in many practical projects, such as subgrade, retaining wall, gravity dam [1]. The dynamic analysis of the medium under the plane strain condition has caused many scholars' attention in the field of earthquake engineering, soil mechanics, geophysics and so on. Sneddon[2] first studied the dynamic response of the elastic half space soil in the condition of plane strain under uniform moving line loads. Lefeuve-Mesgouez et al.[3] used potential function and the Fourier transform to solve the dynamic response problems of single-phase elastic half space under plane strain state in the vicinity of a high-speed moving harmonic strip load. Honglei SUN [4] used the semi analytical method to solve the dynamic response of the transversely isotropic poroelastic half-space soil medium. Fengxi Zhou [5] used Fourier transform to obtain dynamic response problems of analytic expression under harmonic loads in isotropic saturated soil foundation. However, the soil has obvious anisotropy in the process of deposition. In addition, Mengxi Zhang [6] believes that the reinforced soil also has obvious anisotropy. The reinforced soil has been widely used in highway, port, water conservancy, railway and other civil engineering in recent years. The displacement function of the orthotropic elastic plane static problem was derived by Hanzhong Xu [7]. Longzhi Jiang [8] used the integral transform method to deduced the fundamental solutions for plane orthogonal finite foundation subjected to a concentrated load acted in any direction.

Based on the above research, the dynamic equations are established according to the stress-strain relationship of the plane strain problem in rectangular coordinate system. The initial conditions and boundary conditions are introduced, and the integral solutions of the orthotropic semi-infinite medium under harmonic loads are obtained by using Fourier transform and inverse transform. The time domain solution of the displacement and stress components at any moment in a semi-infinite medium with the orthogonal anisotropic media is given.

\section{Dynamic equations and its solution in rectangular coordinate system}

\subsection{Dynamic Equations}

As shown in Fig.1, harmonic strip loads are applied on orthogonal anisotropic half space area. 


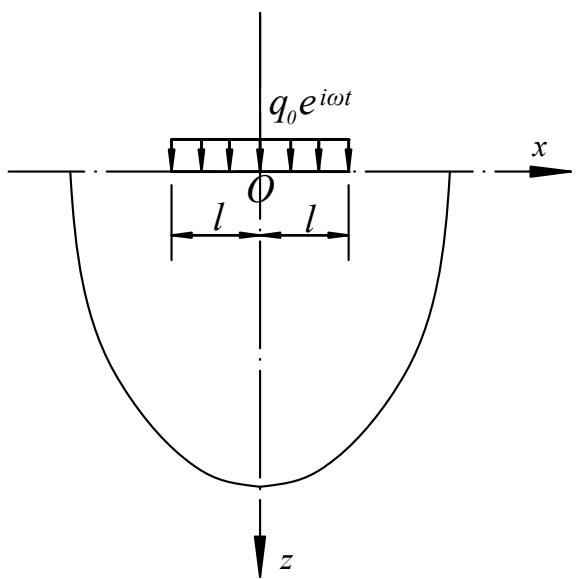

Fig.1 Load distribution on the half-space

$$
\left.\begin{array}{l}
q_{z}(x, t)=q_{z}(x) e^{i \omega t}=q_{0} e^{i \omega t} \quad(-l \leqslant x \leqslant l) \\
q_{x}(x, t)=0 \quad(-\infty \leqslant x \leqslant \infty)
\end{array}\right\} .
$$

The soil in the plane perpendicular to the $x-z$ direction is discredited as infinite distribution, so the problem is simplified to plane strain problem. Rectangular coordinate system is used. Without considering the physical effects, plane strain equilibrium differential equations are

$$
\left.\begin{array}{c}
\frac{\partial \sigma_{x}}{\partial x}+\frac{\partial \tau_{z x}}{\partial z}=\rho \frac{\partial^{2} u_{x}}{\partial t^{2}} \\
\frac{\partial \tau_{x z}}{\partial x}+\frac{\partial \sigma_{z}}{\partial z}=\rho \frac{\partial^{2} u_{z}}{\partial t^{2}}
\end{array}\right\} .
$$

where $\sigma_{x} 、 \sigma_{z} 、 \tau_{z x}$ are the stress components, $\mathrm{Pa} ; \rho$ is the actual mass densities of the soil, $\mathrm{kg} / \mathrm{m}^{3}$; $u_{x} 、 u_{z}$ is-the solid displacement components, along the $x$ and $z$ directions, respectively, $\mathrm{m}$.

The geometric equations are

$$
\left.\begin{array}{l}
\varepsilon_{x}=\frac{\partial u_{x}}{\partial x}, \varepsilon_{z}=\frac{\partial u_{z}}{\partial z} \\
\gamma_{z x}=\frac{\partial u_{x}}{\partial z}+\frac{\partial u_{z}}{\partial x}
\end{array}\right\} .
$$

where $\varepsilon_{x}, \varepsilon_{z}, \gamma_{z x}$ are the strain components.

The stress- strain relations are

$$
\left.\begin{array}{ll}
\sigma_{x}=c_{11} \varepsilon_{x}+c_{13} \varepsilon_{z}, & \sigma_{y}=c_{12} \varepsilon_{x}+c_{23} \varepsilon_{z} \\
\sigma_{z}=c_{13} \varepsilon_{x}+c_{33} \varepsilon_{z}, & \tau_{z x}=c_{55} \gamma_{z x}
\end{array}\right\} .
$$

where $c_{i j}$ are elastic constants, Pa.

Substituting Eqs.3 and 4 into Eq.2 gives

$$
\left.\begin{array}{l}
c_{11} \frac{\partial^{2} u_{x}}{\partial x^{2}}+\left(c_{13}+c_{55}\right) \frac{\partial^{2} u_{z}}{\partial x \partial z}+c_{55} \frac{\partial^{2} u_{x}}{\partial z^{2}}=\rho \frac{\partial^{2} u_{x}}{\partial t^{2}} \\
c_{55} \frac{\partial^{2} u_{z}}{\partial x^{2}}+\left(c_{13}+c_{55}\right) \frac{\partial^{2} u_{x}}{\partial x \partial z}+c_{33} \frac{\partial^{2} u_{z}}{\partial z^{2}}=\rho \frac{\partial^{2} u_{z}}{\partial t^{2}}
\end{array}\right\} .
$$

Eqs.5 are the dynamic response control equations of the orthogonal anisotropic medium under dynamic loads.

\subsection{A General Solution of the Displacement and Stress Components}

For the dynamic response under harmonic loads, there is a direct correspondence between the dynamic responses of frequency domain and time domain. Displacement components $u_{x} 、 u_{z}$ and 
stress components $\sigma_{x} 、 \sigma_{z} 、 \tau_{z x}$ have $e^{i \omega t}$ factor. Therefore, in order to write conveniently, the integral form solutions and corresponding boundary conditions omit the $e^{i \omega t}$ factor.

To solve Eqs.5, we use a spatial Fourier transform defined by

$$
\bar{f}(\xi, z)=\int_{-\infty}^{+\infty} f(x, z) e^{-i \xi x} d x .
$$

In the transform domain, the boundary conditions are as follows

$$
\begin{aligned}
& \lim _{|x| \rightarrow \infty} u_{x}(x, z, t)=0, \lim _{|x| \rightarrow \infty} u_{z}(x, z, t)=0 . \\
& \lim _{|x| \rightarrow \infty} \frac{\partial u_{x}(x, z, t)}{\partial x}=0, \lim _{|x| \rightarrow \infty} \frac{\partial u_{z}(x, z, t)}{\partial x}=0 .
\end{aligned}
$$

Using Eqs. 6 and 7 to transform Eqs. 5 with respect to variable $x$ gives in matrix form as

$$
\left[\begin{array}{cc}
A_{11} & 0 \\
0 & A_{22}
\end{array}\right] \frac{\partial^{2}}{\partial z^{2}}\left[\begin{array}{l}
\overline{u_{x}} \\
\overline{u_{z}}
\end{array}\right]+\left[\begin{array}{cc}
0 & B_{12} \\
B_{12} & 0
\end{array}\right] \frac{\partial}{\partial z}\left[\begin{array}{l}
\overline{u_{x}} \\
\overline{u_{z}}
\end{array}\right]+\left[\begin{array}{cc}
D_{11} & 0 \\
0 & D_{22}
\end{array}\right]\left[\begin{array}{l}
\overline{u_{x}} \\
\overline{u_{z}}
\end{array}\right]=0 \text {. }
$$

where $A_{11}=-c_{55}, B_{12}=-\left(c_{13}+c_{55}\right) i \xi, D_{11}=c_{11} \xi^{2}-\rho \omega^{2}, D_{22}=c_{55} \xi^{2}-\rho \omega^{2}$.

The solution of Eq. 8 is

$$
\left[\begin{array}{ll}
\overline{u_{x}} & \overline{u_{z}}
\end{array}\right]^{T}=\left[\begin{array}{ll}
G_{1} & G_{2}
\end{array}\right]^{T} e^{\lambda z} \text {. }
$$

And substituting Eq.9 into Eq.8 gives the following characteristic equation as

$$
a_{1} \lambda^{4}+a_{2} \lambda^{2}+a_{3}=0 \text {. }
$$

where $a_{1}=A_{11} A_{22}, a_{2}=A_{11} D_{22}+A_{22} D_{11}-B_{12}{ }^{2}, a_{3}=D_{11} D_{22}$.

Four roots of complex coefficient Eq.10 are $\pm \lambda_{1}, \pm \lambda_{2}$.

$$
\lambda^{2}=\frac{1}{2 a_{1}}\left(-a_{2} \pm \sqrt{a_{2}{ }^{2}-4 a_{1} a_{3}}\right) \quad\left(\operatorname{Re}\left[\lambda_{j}\right] \geqslant 0,(j=1,2)\right) .
$$

where $\lambda_{1}^{2}$ and $\lambda_{2}^{2}$ are two unequal complex numbers, then the solutions of matrix Eq.8 are

$$
\left.\begin{array}{l}
\overline{u_{x}}=\sum_{j=1}^{2} \alpha_{j}\left(E_{j} e^{\lambda_{j} z}-F_{j} e^{-\lambda_{j} z}\right) \\
\overline{u_{z}}=\sum_{j=1}^{2}\left(E_{j} e^{\lambda_{j} z}+F_{j} e^{-\lambda_{j} z}\right)
\end{array}\right\} .
$$

where $\alpha_{j}=-\frac{A_{22} \lambda_{j}^{2}+D_{22}}{B_{12} \lambda_{j}}$.

Fourier transforming Eqs. 4 with respect to variable $x$ and Eqs.3 and 12 being substituted gives

$$
\left.\begin{array}{l}
\overline{\sigma_{x}}=c_{11} i \xi \sum_{j=1}^{2} \alpha_{j}\left(E_{j} e^{\lambda_{j} z}-F_{j} e^{-\lambda_{j} z}\right)+c_{13} \sum_{j=1}^{2} \lambda_{j}\left(E_{j} e^{\lambda_{j} z}-F_{j} e^{-\lambda_{j} z}\right) \\
\overline{\sigma_{y}}=c_{12} i \xi \sum_{j=1}^{2} \alpha_{j}\left(E_{j} e^{\lambda_{j} z}-F_{j} e^{-\lambda_{j} z}\right)+c_{23} \sum_{j=1}^{2} \lambda_{j}\left(E_{j} e^{\lambda_{j} z}-F_{j} e^{-\lambda_{j} z}\right) \\
\overline{\sigma_{z}}=c_{13} i \xi \sum_{j=1}^{2} \alpha_{j}\left(E_{j} e^{\lambda_{j} z}-F_{j} e^{-\lambda_{j} z}\right)+c_{33} \sum_{j=1}^{2} \lambda_{j}\left(E_{j} e^{\lambda_{j} z}-F_{j} e^{-\lambda_{j} z}\right) \\
\overline{\tau_{z x}}=c_{55}\left[\sum_{j=1}^{2} \alpha_{j} \lambda_{j}\left(E_{j} e^{\lambda_{j} z}+F_{j} e^{-\lambda_{j} z}\right)+i \xi \sum_{j=1}^{2}\left(E_{j} e^{\lambda_{j} z}+F_{j} e^{-\lambda_{j} z}\right)\right]
\end{array}\right\}
$$

By using the Fourier inverse transform technique to Eqs.12 and 13, an analytical solution of dynamic response is obtained.

For orthotropic half space, vibration transmission is attenuated.

$$
\lim _{|z| \rightarrow \infty} u_{x}(x, z, t)=\lim _{|z| \rightarrow \infty} u_{z}(x, z, t)=0 \text {. }
$$


Therefore, in the integral form of the general solution of the displacement and stress components, there is

$E_{j}=0$.

As shown in figure 1, the corresponding stress boundary conditions are

$$
\left.\begin{array}{ll}
\sigma_{z}(x, 0, t)=-q_{0} e^{i \omega t} & (-l \leqslant x \leqslant l) \\
\tau_{z x}(x, 0, t)=0 & (-\infty \leqslant x \leqslant \infty)
\end{array}\right\} .
$$

By applying Fourier transformation to Eqs.16, boundary conditions can be given as

$$
\left.\begin{array}{l}
\overline{\sigma_{z}}(\xi, 0)=-\frac{2 q_{0}}{\xi} \sin (\xi l) \\
\overline{\tau_{z x}}(\xi, 0)=0
\end{array}\right\} .
$$

And substituting Eqs.15 and 17 into Eqs.13 gives

$$
\left.\begin{array}{l}
F_{1}=\frac{-\left(\alpha_{2} \lambda_{2}+i \xi\right)}{\Delta} \frac{2 q_{0}}{\xi} \sin (\xi l) \\
F_{2}=\frac{\left(\alpha_{1} \lambda_{1}+i \xi\right)}{\Delta} \frac{2 q_{0}}{\xi} \sin (\xi l)
\end{array}\right\} .
$$

where, $\Delta=-\left(c_{13} i \xi \alpha_{1}+c_{33} \lambda_{1}\right)\left(\alpha_{2} \lambda_{2}+i \xi\right)+\left(c_{13} i \xi \alpha_{2}+c_{33} \lambda_{2}\right)\left(\alpha_{1} \lambda_{1}+i \xi\right)$.

Substituting Eqs.15 and 18 into Eqs.13, we can observe the displacement and stress components. Then analytical solutions of dynamic response are obtained by using the Fourier inverse transform technique to all components. Here only expression of the vertical displacement is given.

$$
u_{z}=\frac{q_{0} e^{i \omega t}}{\pi} \int_{-\infty}^{+\infty} \frac{\sin (\xi l)}{\Delta \xi}\left[-\left(\alpha_{2} \lambda_{2}+i \xi\right) e^{-\lambda_{1} z}+\left(\alpha_{1} \lambda_{1}+i \xi\right) e^{-\lambda_{2} z}\right] e^{i \xi x} \mathrm{~d} \xi
$$

\section{Numerical results}

Calculation model is shown in Fig. 1. The soil and load parameters of the literature [3] are used. The values are $E=269 \mathrm{MPa}, v=0.257, \eta=0.1, \rho=1550 \mathrm{~kg} / \mathrm{m}^{3}, l=0.75 \mathrm{~m}, q_{0}=4 \pi / 3 \mathrm{~N} / \mathrm{m}^{2}$. According to the principle of this paper, the numerical analysis is employed by the MATLAB software. The vertical displacement on the surface for the two frequencies 8 and $64 \mathrm{~Hz}$ is shown in Fig. 2. $\left|u_{z}\right|$ means vertical displacement amplitude in Fig. 2. As can be seen, the calculation results obtained by the present approach are in good agreement with the literature [3], which verifies the correctness of this method.

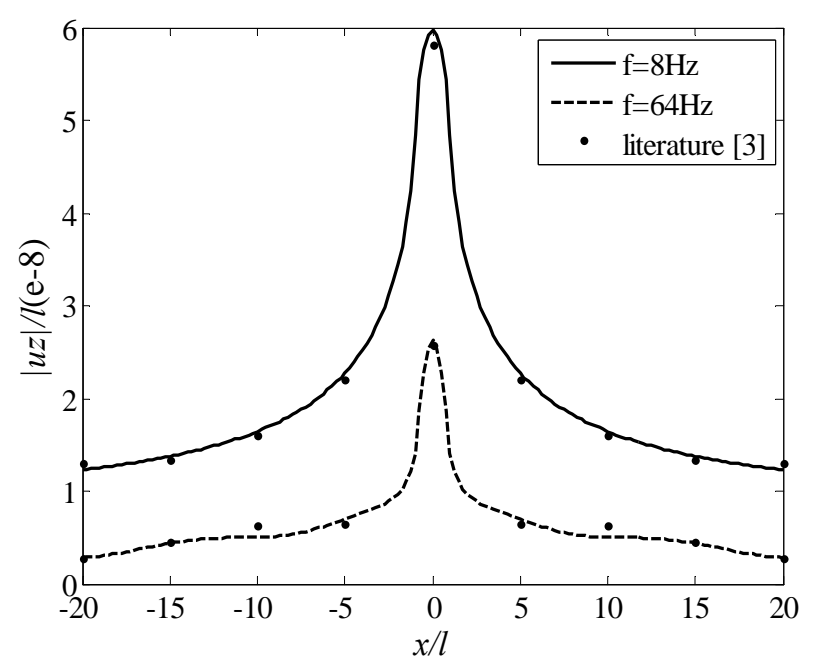

Fig. 2 Contrast of calculation results 
The appropriate parameters of soil are selected, the displacement of each point of soil is calculated and the corresponding changing rules to the parameters are discussed. Calculation parameters are as follows: $c_{11}=39.4 \mathrm{GPa}, c_{13}=5.8 \mathrm{GPa}, c_{55}=4 \mathrm{GPa}, c_{33}=k c_{11}, \rho=1815 \mathrm{~kg} / \mathrm{m}^{3}, l=2 \mathrm{~m}, q_{0}=400 \mathrm{kN} / \mathrm{m}^{2}$. Adjusting the parameter $k$ can get different media.

Vertical displacement curves of different depth are shown in Fig.3 for $k=1 / 3$ and $f=8 \mathrm{~Hz}$. Curves show that the displacement response is gradually decreased with the increase of depth, $\left|u_{z \max }\right| / l=1.286 \times 10^{-4}$ on the surface, $0.5964 \times 10^{-4}$ on depth $10 \mathrm{~m}$. The depth is almost no influence on the vertical displacement of the far distance to surface load center. It is shown in Fig. 4 that non-dimensional vertical displacements on soil surface change in the form of damped oscillation along with the increase of $x$ when $f=10 \mathrm{~Hz}$. We can see that the orthotropic parameters of soil have a great influence on the vertical displacement, $\left|u_{z \max }\right| / l=1.531 \times 10^{-4}$ when $k=1 / 3$, $\left|u_{z \max }\right| / l=0.6931 \times 10^{-4}$ when $k=1$. Fig.5 gives non-dimensional vertical displacement curves on soil surface for different load frequencies when $k=1 / 3$. As can be seen, frequencies have less impact on the maximal displacement amplitude of the center. Vertical displacements change in the form of damped oscillation along with the increase of $x$. The vibration velocity of vertical displacement is accelerated with the increase of harmonic load frequencies.

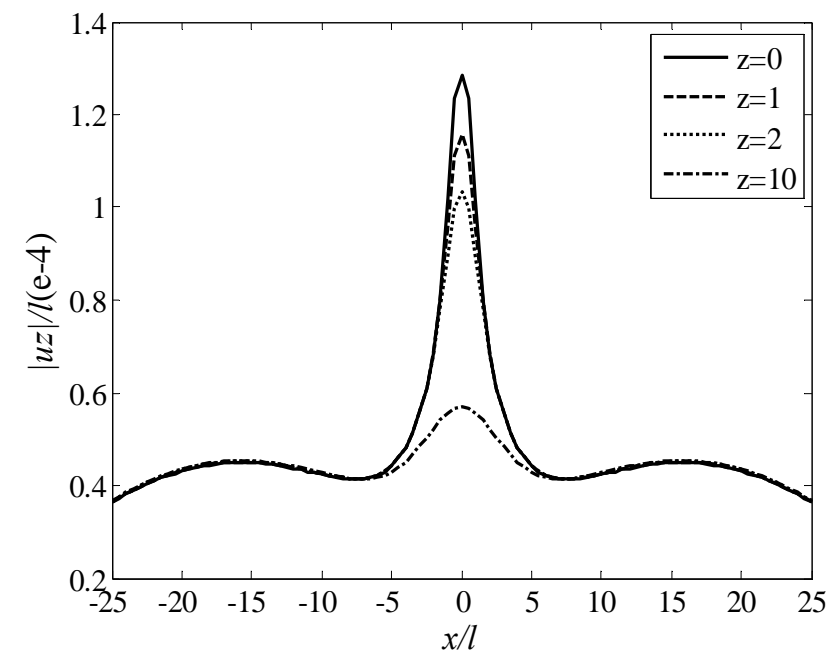

Fig. 3 Non-dimensional vertical displacement curves for different depth, $k=1 / 3, f=8 \mathrm{~Hz}$

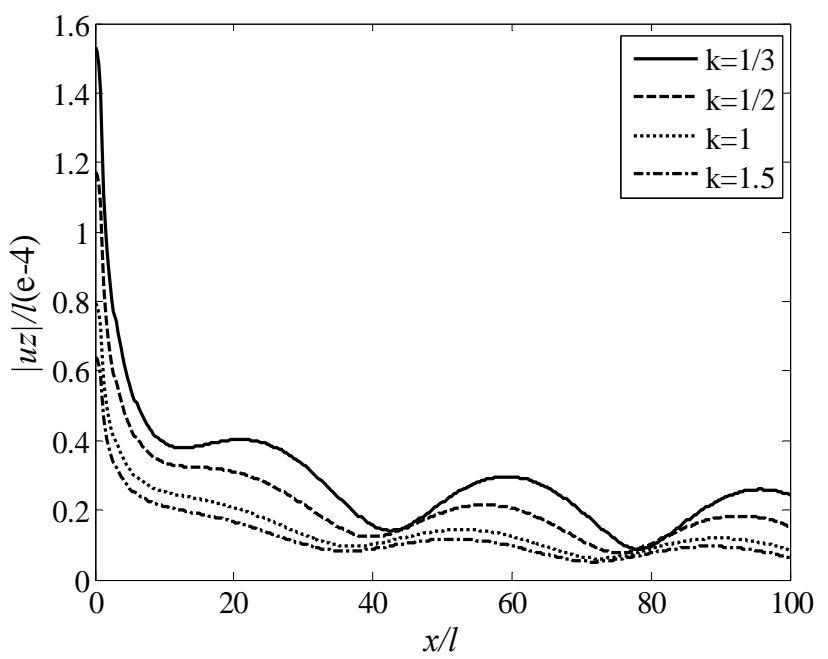

Fig. 4 Non-dimensional vertical displacement curves on soil surface, $f=10 \mathrm{~Hz}$

\section{Conclusions}

This paper studies the dynamic response of the orthogonal anisotropic medium plane strain problem under harmonic loads. The general solutions of the integral form of the displacement and stress 
components are given. The numerical results show that: (1) with the increase of depth, the displacement response is gradually reduced, and the depth has almost no effect on the vertical displacement of the far distance to surface load center. (2) The orthogonal anisotropic parameters of soil have a great influence on vertical displacement amplitude. (3)The maximum displacement decreases and the vibration velocity of vertical displacement accelerates with the increase of harmonic load frequencies. The results of this paper provide a theoretical basis for the further study of the dynamic analysis of orthotropic soil and structure.

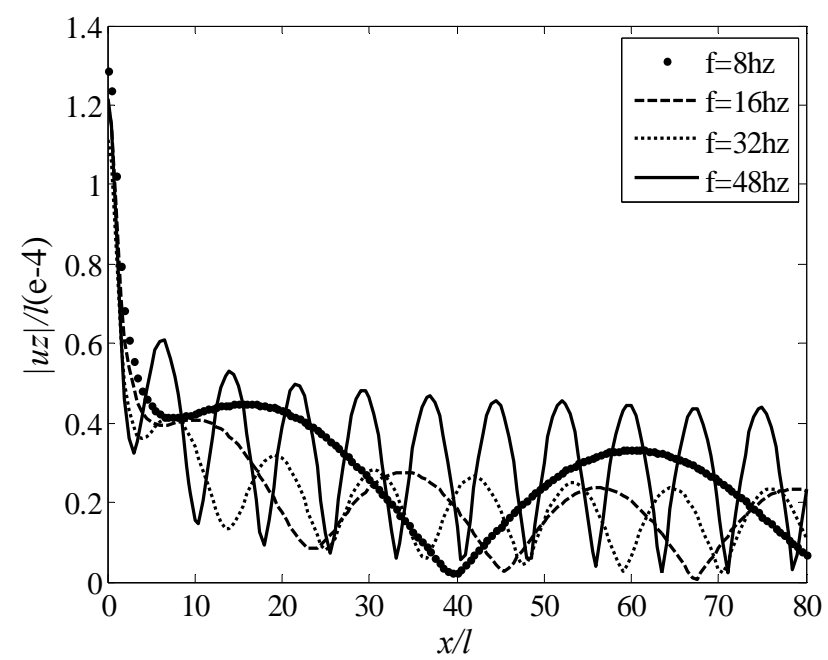

Fig. 5 Non-dimensional vertical displacement curves on soil surface for different load frequencies, $k=1 / 3$

\section{Acknowledgements}

This work was financially supported by Foundation for University Key Teacher by the Ministry of Education of Henan Province (2015GGJS-277) and Science and Technology Planning Project of Henan Province (08212360056 and112300410286).

\section{References}

[1] Zhilun Xu: Elasticity (4th edition)( Higher Education Press, Chinese2006).

[2] Ian. N. Sneddon: Rendiconti del Circolo Matematico di Palermo, vol.1, no. 1, pp. 57-62(1952).

[3] G.Lefeuve-Mesgouez, D.Le HOUÉdec, A.T.Peplow: Journal of Sound and Vibration, vol. 231, no. 5, pp. 1289-1309(2000).

[4] Honglei SUN, Yuan-qiang CAI, Chang-jie XU: Journa1 of Zhejiang University(Engineering Science), vol. 40, no. 8, pp. 1382-1387(2006)(in Chinese).

[5] Fengxi Zhou,Yuanming Lai,Yuanyuan Ren,: Chinese Journal of Solid Mechanics, vol. 34, no. 5, pp. 536-540(2013) (in Chinese).

[6] Mengxi Zhang: Journal of the China Railway Society, vol. 15, no. 3, pp. 84-89(1993) (in Chinese).

[7] Hanzhong Xu: Journal of Hehai University, vol. 20, no. 5, pp. 21-30(1992) (in Chinese).

[8] Longzhi Jiang, Youcheng Wang : Journal of Hefei University of Technology, vol. 14, no. 2, pp. 29-37(1991) (in Chinese). 